\title{
Comparison of Adaptive Filtering Algorithms Based on Time Varying Weighting Factor
}

\author{
Mohammed Abdul Nasar \\ CVR College of Engineering/ECE Department, Hyderabad, India \\ Email: nasar.mohammed@gmail.com
}

\begin{abstract}
In this paper, a Leaky Time Varying Mean Mixed Norm Adaptive algorithm is proposed. The weight drift issue is overcome by minimizing the function defining weighted sum of the LMS and LMF cost functions along with the leakage factor. The weighting factor is applied in time varying environment and made to adapt itself so as to emphasize one cost function over the other based on closeness to the optimum value. The proposed algorithm results are compared with the conventional algorithms and the obtained results show an improvement over the convergence.
\end{abstract}

\section{Index Terms - Adaptive Filters, Fixed Mixed Norm (FMN), Variable Leaky LMMN (VLLMMN)}

\section{INTRODUCTION}

The least mean-square (LMS) [3] algorithm and the least mean-fourth (LMF) [4] algorithm can be generalized as minimization of the mean- $k t h$ error function, that is $J_{n}=E\left[e_{n}^{k}\right], k$ is some positive integer, when $k=2$ and $k=4$ are substituted above, the equation results in the LMS and LMF algorithms [1,2] respectively.

As can be seen from Figure (1), adaptive filters can be used to solve the problem of system identification, wherein a model is created to overcome the uncertainties in the system when its parameters are unknown to us and may be time varying. In such cases same input $x_{n}$ is being fed to both the adaptive filter and the system under modeling. The difference between them $d_{n}$ and the responses of the adaptive filter $y_{n}$ and the system are then compared i.e. the error $\mathrm{e}_{\mathrm{n}}$ is used to adjust the parameters of the filter. With the increase in the number of iterations, the parameters of the adaptive filter reach an optimum value or approach to those of unknown system parameters as it is clearly evident from Figure (1).

In previous work [9], it's been noticed that LMF algorithm performs well compared to the LMS algorithm in the environment which is non-Gaussian. As a variant of the LMS algorithm we find another example of adaptive filter algorithms is the leaky Least Mean Square (leaky LMS) algorithm [10]. So the leaky LMS algorithm was first introduced to overcome the weight drift problem occurring in the LMS adaptive filters which usually occurs due to insufficient excitation of the input. Following the same analysis it's seen that the LMF algorithm also suffers from the weight drift problem under the same conditions as LMS algorithm. Making use of the best properties of these two algorithms leads us to the LMMN algorithm [5], which is found to provide a better performance in different noise environments like Gaussian and Non-Gaussian compared to either LMS or the LMF. But even LMMN algorithm undergoes a weight drift problem under insufficient excitation of the input signal.

The LMS algorithm which reduces the square of the error is a very well known algorithm in adaptive filtering, while the LMF algorithm which reduces the fourth of the error has been proposed in [4] and gained much attention in the literature proposed in [5]-[7]. Both the algorithms are robust to the noise statistics in different environments like Gaussian noise, Uniform noise to name a few, because of which their convergence behaviour are different [4]. From the above discussion it is well understood that the larger gradient of LMF algorithm makes the convergence faster when away from the optimum $\left(e_{n}^{4}>e_{n}^{2}\right.$ for $\left.e_{n}^{2}>1\right)$. But more desirable characteristics in the neighborhood of optimum are obtained by making use of LMS algorithm.

In the literature work the weighted sum of the two performance measures has been utilized and was proposed to combine the advantages of both in the mixed-norm adaptive algorithm [5]. The mixed-norm LMS-LMF adaptive algorithm is defined by the following cost function:

$$
J_{n}=\delta E\left[e_{n}^{2}\right]+(1-\delta) E\left[e_{n}^{4}\right]
$$

Where $\delta \in[0,1]$ controls the mixture of the mixed norm algorithm i.e. a value of $\delta=1$ in the above equation results in LMS algorithm whereas a value of $\delta=0$ leads to the LMF algorithm convergence behavior respectively. $e_{n}$ is the error between the adaptive filter and the unknown system output and is usually defined as

$$
e_{n}=y_{n}-x_{n} w_{n}
$$

where $y_{n}$ is the desired value, $w_{n}$ is the filter coefficient 
of the adaptive filter being employed, and $x_{n}$ is the input vector.

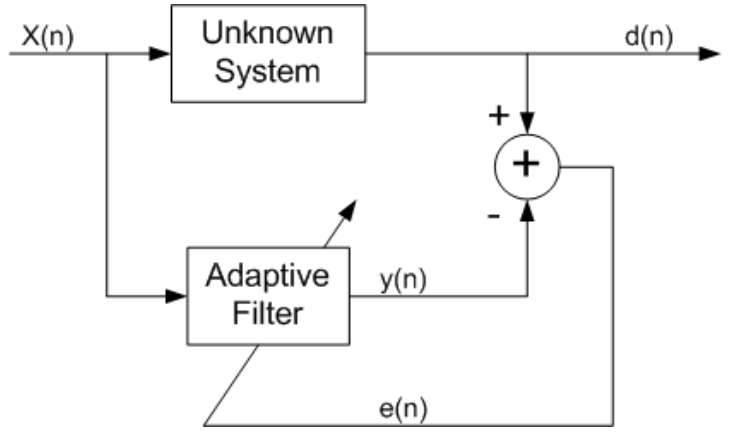

Figure1: Block Diagram of Adaptive System Identification.

So the combination of the conventional LMS and LMF algorithms give LMMN algorithm. But at some times the LMMN algorithm continues the error controlling process even if the input is not present i.e. what we call as weight drift problem in case of adaptive algorithms. To overcome this problem of weight drifting a leakage factor is introduced in the algorithm giving rise to Leaky LMMN algorithm [9].

The LMMN cost function is modified a little bit to obtain Leaky LMMN by introducing a leakage parameter $\varepsilon$ in the same way as was done in the literature for the case of the leaky LMS [8]. The term leakage stems out from the fact that, unlike the conventional LMS, where weights remain stationary in case of stalling, in Leaky LMMN the weights "leak out" in case of stalling occurs i.e. the input sequence becomes zero. Therefore, the cost function that is required to minimize the error is given as

$$
J(w)=\varepsilon\|w\|^{2}+\left\{\delta E\left[e_{n}^{2}\right]+(1-\delta) E\left[e_{n}^{4}\right]\right\}
$$

Where $J$ is the cost function in finding out the optimum weight vector $\delta$ controls the mixture of the mixed norm algorithm related to the error vector $e_{n}$ and $\varepsilon$ is the leakage factor which mitigates the weight drift problem occurring in the mixed norm algorithm. Following the above discussion the normalized weight error in the case of Leaky LMMN can be obtained from [9] as

$\left\|\tilde{\mathrm{w}}_{n+1}\right\|^{2}=(1-\mu \varepsilon)^{2}\left\|\tilde{w}_{n}\right\|^{2}+\mu^{2}\left\|u_{n}\right\|^{2} v_{n}^{2}$

So that $\left\|\tilde{w}_{n}\right\|^{2}$ remains bounded for $0<\mu \varepsilon<1$.

\section{PROPOSED ALGORITHM}

The algorithm defined in equation (3) works when the "mixing" parameter or weighting factor is fixed and is does not consider the leakage in the time varying environment and is usually predetermined. In this paper, a self-adapting time variable weighting factor is considered that brings our proposed algorithm to the LMF algorithm when the coefficient vector is away from the optimal value and on the other hand emphasizes the LMS algorithm when the coefficient vector is close to the optimum. Here a time varying weighting factor is proposed to allow the algorithm to adapt itself to the changing input conditions and is denoted as variable $\delta_{n}$. This factor is then updated every iteration so it is large when we are away from the optimum and decreases as we approach towards the optimum.

In the derivation of the proposed algorithm, modified variable step size (MVSS) algorithm is being used as proposed in [7] to update the weighting factor $\delta_{n}$. The proposed variable weight leaky mixed-norm LMS-LMF algorithm required to minimize the performance measure can be thus defined as:

$$
J(w)=\varepsilon\|w\|^{2}+\left\{\delta_{n} E\left[e_{n}^{2}\right]+\left(1-\delta_{n}\right) E\left[e_{n}^{4}\right]\right\}
$$

where $\delta_{n}$ is a time varying parameter updated according to:

$$
\delta_{n+1}=\alpha \delta_{n}+\gamma p^{2}(n)
$$

and

$$
p(n)=\beta p(n-1)+(1-\beta) e(n) e(n-1)
$$

The parameters $\alpha$ and $\beta$, are the exponential weighting parameters governing the average time constant and are usually confined to the interval $[0,1]$, i.e., these parameters are responsible for the quality of estimation, and $\gamma>0$. It can be observed from the algorithm defined in equation (1) that it can be restored efficiently when $\alpha=1$ and $\gamma=0$, i.e., $\delta_{n}$ is chosen to have a fixed value.

If the constraints $\alpha$ and $\gamma$ as defined in equation (5) are given, than making use of uni-modal character the cost function defined in equation (3) can be preserved.

Following the discussion above it motivates for the development of the proposed algorithm that recursively adjusts the coefficients of a leaky system which is expressed in the following form, i.e., update equation of the variable leaky LMMN can be written as:

$$
w_{n+1}=(1-\mu \varepsilon) w_{n}+\mu e_{n}\left\{\delta_{n}+\left(1-\delta_{n}\right) e_{n}^{2}\right\} x_{n}
$$


where $\mu$ is the step size chosen small enough for the convergence of the algorithm to take place and the sufficient condition for the convergence of the proposed algorithm in the mean square sense can be found out in the same way as the approach used for the mean convergence on the step size and is usually is governed by the equation

$0<\mu<\frac{2}{\varepsilon+\left[E\left[\delta_{n}\right]+3 E\left[\left(1-\delta_{n}\right)\right]\left(\sigma_{v}^{2}+\vartheta\right) \lambda_{\max }(R)\right]}$

where Where $\lambda_{\max }(R)$ is the largest eigen value of $\mathrm{R}$, $\sigma_{v}^{2}$ is the input noise power and $E\left[\delta_{n}\right]$ is the mean of the mixing parameter.

From Equation (6), $p(n)$ can be set recursively in the following form:

$$
p(n)=(1-\beta) \sum_{i=0}^{n-1} \beta^{i} e(n-i) e(n-i-1)
$$

and therefore

$$
p^{2}(n)=(1-\beta)^{2} \sum_{i=0}^{n-1} \sum_{j=0}^{n-1} \beta^{i} \beta^{j} e(n-i) e(n-i-1) e(n-j) e(n-j-1)(10)
$$

Making use of the above set of recursive equations an expression for the mean of the mixing parameter, namely $E\left[\delta_{n}\right]$ can be obtained as

$$
\begin{aligned}
& E\left[\delta_{n+1}\right]=\alpha E\left[\delta_{n}\right]+\gamma(1-\beta)^{2} \sum_{i=0}^{n-1} \beta^{2 i} \\
& \times E\left[e^{2}(n-i)\right] \\
& \times E\left[e^{2}(n-i-1)\right]
\end{aligned}
$$

In the above Equation, its been assumed that the algorithm has converged, and in this case the samples of the error e(n) can be assumed uncorrelated, i.e., $E[e(n-i) e(n-j)] \simeq 0, \forall(i \neq j)$.

Also, the mean-square error (MSE) can be set into the following:

$$
E\left[e^{2}(n)\right]=E\left[e_{a}^{2}(n)\right]+\sigma_{n}^{2}
$$

where $\sigma_{n}^{2}$ and $E\left[e_{a}^{2}(n)\right]$ are the noise power and the excess MSE, respectively.

\section{SIMULATION RESULTS}

The signal to be processed is worked out for both Gaussian and Uniform noise environment and then the time Variable Leaky Least Mean Mixed Norm
(VLLMMN) algorithm is compared to the Fixed MixedNorm (FMN) LMS-LMF algorithm in terms of faster convergence. The input signal $x_{n}$ which is not known to the system and to the adaptive filter is considered to be \pm 1 sequence and the channel used is $w_{o}=[0.3482,0.8704,0.3482]^{T}$.

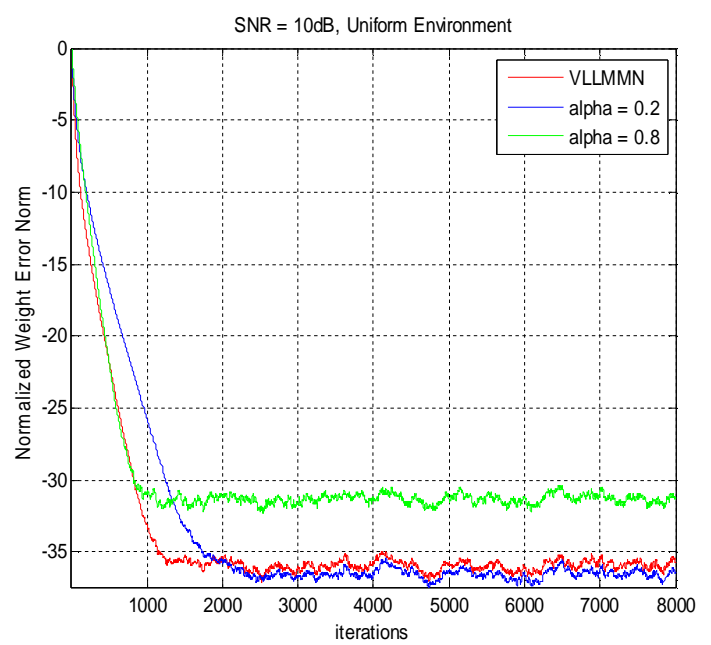

Figure 2: Behaviour of Proposed and the FMN algorithm in Uniform
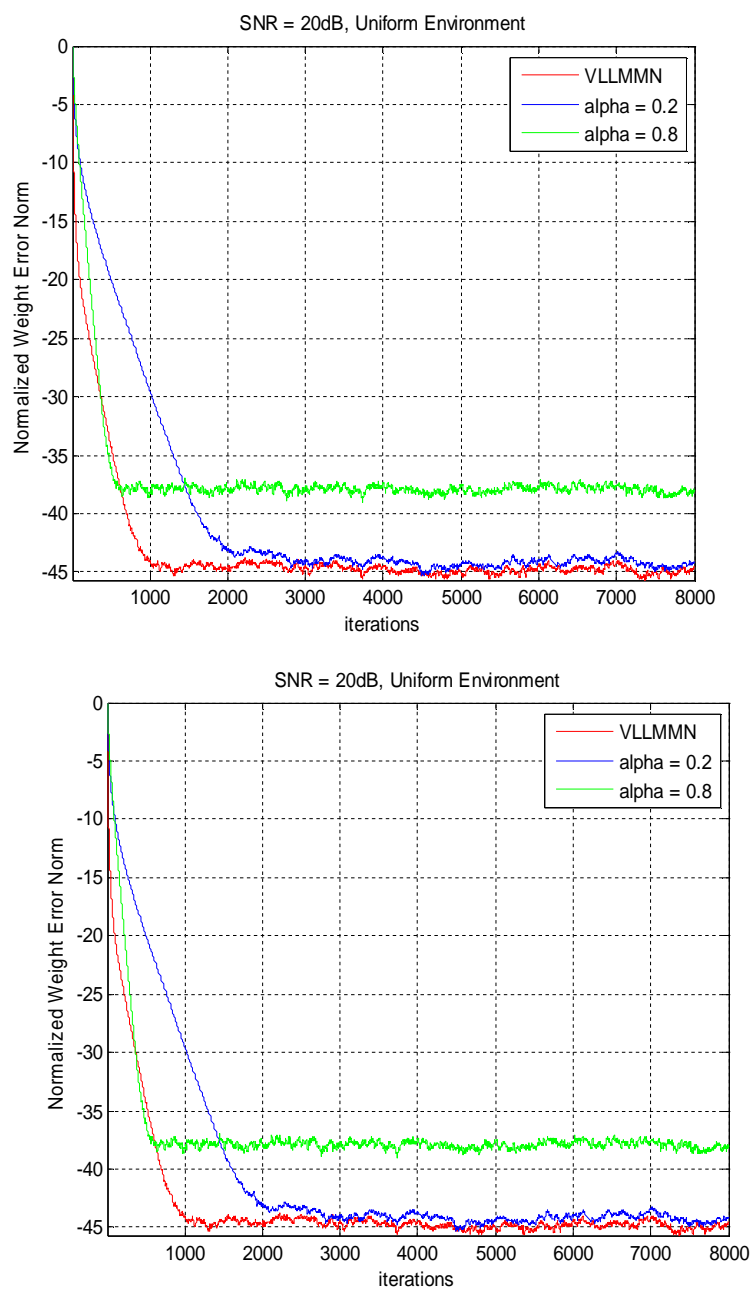

Figure 3: Behaviour of Proposed and the FMN algorithm in Uniform Noise and noise variance 0.2 


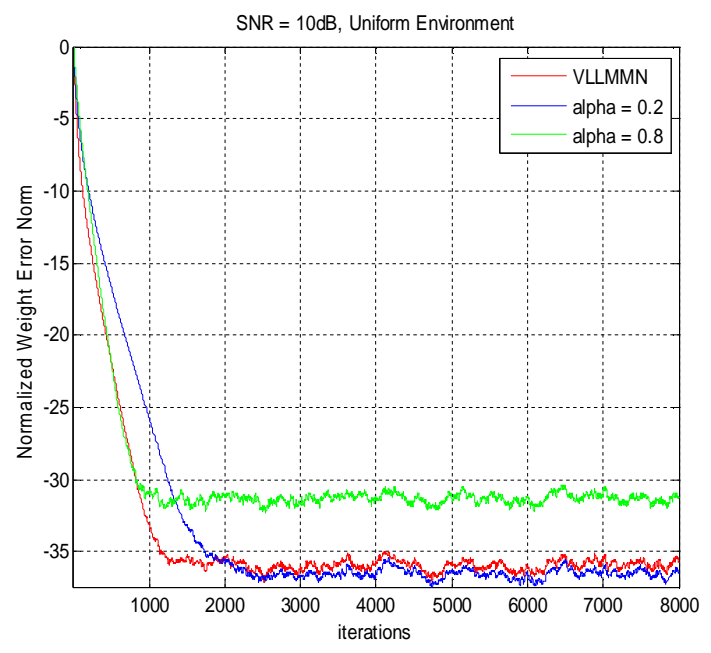

Figure 2: Behaviour of Proposed and the FMN algorithm in Uniform Considering a white input data, where the variance of the regressors is set to unity and maintaining all the parameters as defined above, the simulations in this paper are performed for Uniform and Gaussian noise environments with noise variance i.e Signal to Noise ratio (SNR) value set at $10 \mathrm{~dB}$ and $20 \mathrm{~dB}$ respectively. The performance measure considered is the normalized weight error norm $10 \log _{10}\left\|w_{n}-w_{o}\right\|^{2} /\left\|w_{o}\right\|^{2}$ with the number of taps set to 5 . The additive noise is a zero mean and has a Gaussian distribution. Results are obtained by averaging 8000 samples over 100 independent runs.

Now comparing the time varying algorithm to the fixed mixed-norm algorithm for different values of mixing parameter $\delta$.

From the above figures the fixed mixed norm algorithm is considered with $\delta=0.8$ and $\delta=0.2$. The FMN algorithm with $\delta=0.8$ behaves almost similarly to the LMS algorithm whereas the FMN algorithm with $\delta=0.2$ emphasizes the approach towards the LMF algorithm. The superior performance of the proposed algorithm compared to the conventional algorithms can be observed from the Figures (2-5) where in the proposed Variable Leaky LMMN algorithm results outperform the two versions of the FMN algorithm. This is a result of the fact that the mixing parameter for the proposed algorithm is time varying, which accommodates itself according to changes in the environment.

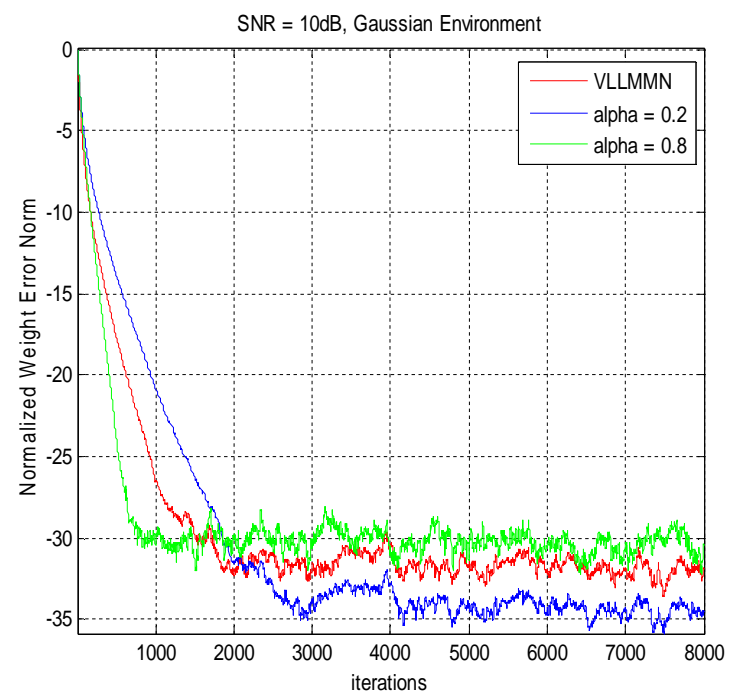

Figure 4: Behaviour of Proposed and the FMN algorithm in Gaussian Noise and noise variance 0.1

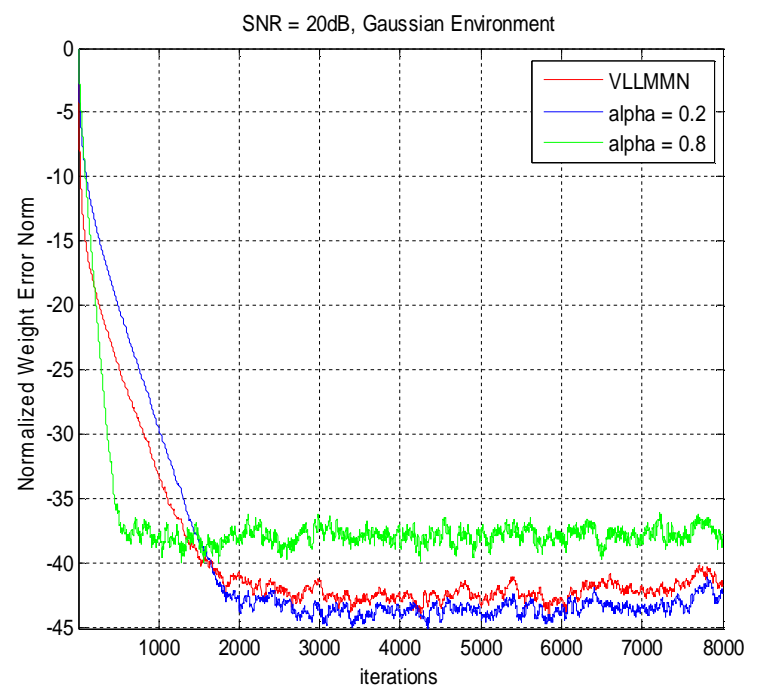

Figure 5: Behaviour of Proposed and the FMN algorithm in Gaussian Noise and noise variance 0.2

It is clearly noticeable from figures (2-5) that the proposed Variable Leaky Least Mean Mixed Norm Algorithm (VLLMMN) algorithm totally overcomes the FMN algorithm. With $\delta=0.8$, it is observed from the simulations that the later has the same convergence rate as that of the proposed algorithm but results in larger excess steady state MSE. On the other hand, when $\delta=0.2$ is considered for the FMN algorithm as compared with the proposed VLLMMN algorithm, it results in the same excess steady state MSE as the proposed algorithm, but the later has a faster speed of convergence. 


\section{CONCLUSIONS}

A time varying mixed-norm algorithm i.e Variable Leaky Least Mean Mixed Norm Algorithm (VLLMMN) is proposed herein, where a combination of the LMS and the LMF algorithms is incorporated using the concepts of variable step size LMS adaptation. It is found to outperform both the LMS and the LMF algorithms in the time varying environment. A bound on the step size to ensure convergence in the mean is also provided. Finally, the consistency and the performance of the proposed algorithm are supported by the simulation results.

\section{REFERENCES}

[1] A.H. Sayed, Fundamentals of Adaptive Filtering. New York, NY:Wiley Interscience, 2003.

[2] S. Haykin, Adaptive Filter Theory, Prentice- Hall, Englewood Cliffs, NJ, 1991.

[3] B. Widrow, J. M. McCool, M. G. Larimore, and C. R. Johnson, "Stationary and Nonstationary Learning Characteristics of the LMS Adaptive Filter," Proc. IEEE, vol. 64, No. 8, pp. 1151-1162, Aug. 1976.

[4] E. Walach and B. Widrow, "The Least Mean Fourth (LMF) Adaptive Algorithm and its Family," IEEE Trans. Inf. Theory, vol. IT- 30, pp. 275-283, Feb. 1984.

[5] J. A. Chambers, 0. Tanrikulu, and A. G. Constantinides, "Least mean mixed-norm adaptive filtering," Electr. lett., vol. 30, No. 19, pp. 1574-1575, Sep. 1994.

[6] A. Zerguine, C. F. N. Cowan, and M. Bettayeb, "Novel LMS-LMF Adaptive Scheme for Echo Cancellation," Electr. lett., vol. 32, No. 19, pp. 1776-1778, Sep. 1996.

[7] T. Aboulnasr and K. Mayyas, "A Robust Variable Step-Size LMS-Type Algorithm: Analysis and Simulations," IEEE Trans. Signal Processing, vol. SP-45, No. 3, pp. 631-639, March, 1997.

[8] W. Sethares et al, "Parameter Drift in LMS Adaptive Filters", IEEE Trans. Accoustics, Speech and Signal Processing, Vol. ASSP-34, No.4, August 1986

[9] Mohammed Abdul Nasar, "The Leaky Least Mean Mixed Norm Algorithm" ISSN 2277-3916, CVR Journal of Science and Technology, Volume 5, December 2013.

[10] R.D. Gitlin, H.C. Meadors and H.B. Weinstein, "The Tap Leakage Algorithm: An Algorithm for the Stable Op-eration of a Digitally Implemented Fractionally-Spaced Adaptive Equalizer,” Bell Sys. Tech. Journal, vol. 61, pp. 1817-1840, Oct. 1982. 